\title{
QUASICONFORMAL EXTENSION OF HOLOMORPHIC MAPPINGS OF A BALL IN $C^{n}$
}

\author{
BY J. A. PFALTZGRAFF ${ }^{1}$
}

Communicated by F. W. Gehring, October 16, 1973

Let $C^{n}$ denote the space of $n$ complex variables $z=\left(z_{1}, \cdots, z_{n}\right)$ with Euclidean norm $\|z\|$. The open unit ball $\left\{z \in C^{n}:\|z\|<1\right\}$ is denoted by $B^{n}$. We consider holomorphic functions $f(z)=\left(f_{1}(z), \cdots, f_{n}(z)\right), z \in B^{n}$, from $B^{n}$ into $C^{n}$. The second derivative of such a function is a symmetric bilinear operator, $D^{2} f(z)(\cdot, \cdot)$ on $C^{n} \times C^{n}$, and $D^{2} f(z)(z, \cdot)$ is the linear operator obtained by restricting $D^{2} f(z)$ to $z \times C^{n}$, with matrix representation

$$
D^{2} f(z)(z, \cdot)=\left(\sum_{m=1}^{n} \frac{\partial^{2} f_{k}(z)}{\partial z_{j} \partial z_{m}} z_{m}\right), \quad 1 \leqq j, k \leqq n .
$$

A locally biholomorphic mapping $f(z)$ from a domain $G \subset C^{n}$ into $C^{n}$ is said to be $K$-quasiconformal in $G$ if $\|D f(z)\|^{n} \leqq K|\operatorname{det} D f(z)|, z \in G$, where \|\| denotes the standard operator norm $\|A\|=\sup \{\|A w\|:\|w\| \leqq 1\}$, $A \in \mathscr{L}\left(C^{n}\right)$.

The purpose of this note is to announce the following $n$-dimensional $(n \geqq 1)$ generalizations of one-dimensional results due to J. Becker [1].

THEOREM. Let $f(z)$ with $D f(0)=I$ be locally biholomorphic in $B^{n}$ and satisfy

$$
\left(1-\|z\|^{2}\right)\left\|(D f(z))^{-1} D^{2} f(z)(z, \cdot)\right\| \leqq c, \quad z \in B^{n} .
$$

If $c \leqq 1$ then $f$ is univalent in $B^{n}$ and

$$
\|z\| /(1+c\|z\|)^{2} \leqq\|f(z)\| \leqq\|z\| /(1-c\|z\|)^{2}, \quad z \in B^{n} .
$$

If $f$ is $K$-quasiconformal in $B^{n}$ and $c<1$ then $f$ is univalent and continuous in the closed ball, $\bar{B}^{n}$, and $f$ can be extended to a quasiconformal homeomorphism of $R^{2 n}$ onto $R^{2 n}$.

For $n=1$, (1) is $\left|z f^{\prime \prime}(z)\right| f^{\prime}(z) \mid \leqq c /\left(1-|z|^{2}\right)$, the local biholomorphy implies $f$ is 1-quasiconformal in $B^{1}$, and our theorem coincides with

AMS (MOS) subject classifications (1970). Primary 32A10; Secondary 30A36, $30 \mathrm{~A} 60$.

${ }^{1}$ Research supported in part by the U.S. Army Research Office-Durham Grant 31-124-72G182E. 
Becker's results. The quasiconformal extension of $f$ is not holomorphic on all of $C^{n}$, but viewed as a mapping of $R^{2 n}$ to $R^{2 n}$, it is ACL, differentiable a.e., and the dilatation is uniformly bounded a.e. (cf. [5, p. 115]). By arguments similar to Becker's, we derive these results from our $n$ dimensional generalization [3] of Pommerenke's theory of subordination chains [4], and from the following lemma.

LEMMA. Let $f(z)$ be locally biholomorphic and $K$-quasiconformal in $B^{n}$. If $f$ satisfies (1) with $c<2$ then

$$
\|D f(z)\|=O\left(1 /(1-\|z\|)^{c}\right), \quad z \in B^{n},
$$

and $f$ has a continuous extension to $\bar{B}^{n}$ that satisfies a Lipschitz condition

$$
\|f(z)-f(w)\| \leqq M\|z-w\|^{1-c}, \quad z, w \in \bar{B}^{n} .
$$

The proof of (2) is fairly elementary, and does not require the use of subordination chains. The proof that (2) implies (3) depends upon $n$ dimensional versions of classical theorems of Hardy and Littlewood [2, pp. 361-363].

Complete proofs of our results and details of the theory of $n$-dimensional subordination chains will be submitted for publication elsewhere [3].

\section{REFERENCES}

1. J. Becker, Löwnersche Differentialgleichung und quasikonform fortsetzbare schlichte Funktionen, J. Reine Angew. Math. 255 (1972), 23-43. MR 45 \#8828.

2. G. M. Goluzin, Geometric theory of functions of a complex variable, GITTL, Moscow, 1952; German transl., VEB Deutscher Verlag, Berlin, 1957; English transl., Transl. Math. Monographs, vol. 26, Amer. Math. Soc., Providence, R.I., 1969. MR 15, 112; 19, 735; 40 \#308.

3. J. A. Pfaltzgraff, Subordination chains, univalence, and quasi-conformal extension of holomorphic mappings in $C^{n}$ (manuscript).

4. Ch. Pommerenke, Über die Subordination analytischer Funktionen, J. Reine Angew. Math. 218 (1965), 159-173. MR 31 \#4900.

5. J. Väisälä, Lectures on n-dimensional quasiconformal mappings, Lecture Notes in Math., vol. 229, Springer-Verlag, Berlin and New York, 1971.

Department of Mathematics, University of North Carolina at Chapel Hill, North Carolina 27514 